\title{
Prospects for vibromechanical spheroidization of metal powders
}

\author{
Alexander Samukov ${ }^{1}$, Margarita Cherkasova², Maksim Kuksov ${ }^{3}$, Sergei Dmitriev ${ }^{4}$ \\ REC "Mekhanobr-Tekhnika”, Saint Petersburg, Russia \\ ${ }^{2}$ Corresponding author \\ E-mail: ${ }^{1}$ samykov_ad@mtspb.com, ${ }^{2}$ cherkasova_mv@mtspb.com, ${ }^{3} k u k s o v \_m p @ m t s p b . c o m$, \\ ${ }^{4}$ dmitrievsv@@mtspb.com
}

Received 19 April 2021; received in revised form 21 May 2021; accepted 28 May 2021 DOI https://doi.org/10.21595/vp.2021.22006

Check for updates

Copyright $(2021$ Alexander Samukov, et al. This is an open access article distributed under the Creative Commons Attribution License, which permits unrestricted use, distribution, and reproduction in any medium, provided the original work is properly cited.

\begin{abstract}
The paper contains a review of the main technologies used for producing metal powders for 3D printing. The prospects are shown for the development of an energy-saving technology for mechanical spheroidization of metal powders using metal processing waste and vibration effects. The results of respective experiments are presented.
\end{abstract}

Keywords: additive manufacturing, manufacture of metal powders, metal processing waste recycling, vibration grinding, ball grinding, metal chips, spheroidization of metal powders.

\section{Introduction}

Metal powders traditionally used in powder metallurgy are generally manufactured by reduction, electrolysis, and melt dispersion; some powders are obtained by mechanical grinding of metal processing waste. The resulting powder particle shape, however, prevents their direct use in $3 \mathrm{D}$ printing, requiring spheroidization of the particles in an additional process.

The term "atomization of particles" is most often used for the spheroidization technologies. These technologies are highly energy-intensive. According to the manufacturers of various types of atomizers (Hermiga, Viga, BluePower, EasyFashion), the average specific energy consumption for metal powder spheroidization is $0.8-1.2 \mathrm{kWh} / \mathrm{kg}$, without the costs of respective finishing to reach the commercial grade.

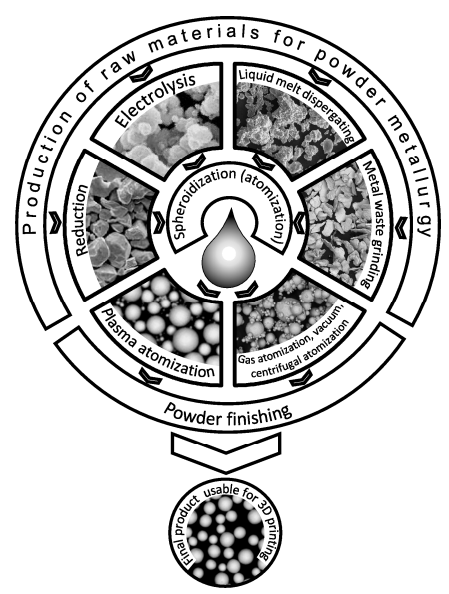

Fig. 1. Common methods for manufacturing metal powders for $3 \mathrm{D}$ printing

Several main industrial technologies for particle atomization are currently available, namely: gas, plasma, vacuum, and centrifugal atomization. These technologies use different equipment sets, but some of them ensure similar characteristics of the atomizer outlet product and require similar finishing processes to reach the desired grade [1].

Gas, vacuum, and centrifugal atomization renders metal powders with a predominantly 
spherical particle shape; however, in the spraying process, individual particles of different diameters tend to stick together, colliding with each other and forming satellites. These particles are separated from the conditioned particles and rejected by various methods, including electrostatic separation [2]. Air classification is used [3] to obtain narrow powder size fractions.

Plasma atomization allows obtaining a higher content of spherical particles, mainly free of satellites, however entails the formation of a large number of submicron particles (less than $5 \mu \mathrm{m}$ ), for which air classification is not feasible and an ultrasonic bath is required [4].

In a simplified form, the process for obtaining raw materials for $3 \mathrm{D}$ printing may be represented in the form of the diagram in Figure 1, summarizing the main manufacturing methods.

When metal powders are obtained from metal processing wastes by mechanical grinding in a ball or vibration mill, non-spherical particles are formed in the particle size range of less than $125(74) \mu \mathrm{m}$ that are mainly lamellar or flaky [9]. Similar results were obtained using a laboratory planetary mill at the Isfahan University of Technology [5].

Therefore, the resulting product cannot be directly used for 3D printing and requires the energy-consuming atomization technologies. However, there is also an alternative approach.

B.P. Fullenwider shows that spherical metal powder particles may be obtained by two-stage grinding of steel chips in a planetary mill [6]. The paper indicates the higher efficiency of a twostage grinding process as compared to a single-stage process when obtaining particles with the size of less than $150 \mu \mathrm{m}$ with an equivalent total grinding time ( 60 hours). The use of $20 \mathrm{~mm}$ balls at the first stage ensures proper crushing of coarse chips to the size of hundreds of microns. When $6 \mathrm{~mm}$ balls are used at the second stage, the particles are further reduced with the formation of a morphology that is close to spherical. The use of $6 \mathrm{~mm}$ balls in the second stage turned out to be more effective than using only $20 \mathrm{~mm}$ balls due to the high frequency of exposure of particles to low forces acting in random directions and the significantly lower maximum deformation depth. The subsequent fabrication of a part using the material obtained has confirmed its suitability for laser sintering.

The National Institute of Technology Rourkela [7] has researched the manufacture of powders from iron, nickel, and chromium in a planetary mill using $8 \mathrm{~mm}$ balls and also indicates the possibility of obtaining spherical particles under certain grinding conditions.

In fact, this line of research facilitates a significant reduction in energy consumption in metal processing waste recycling. After all, the optimal load distribution for the two grinding stages with the correct ball size selection for each stage will ensure the required spherical shape of the finely ground metal powder particles with practically the same energy consumption that has been previously required solely for grinding the chips. This will also eliminate the energy consumption for additional atomization.

When evaluating the research results published by B.P. Fullenwider, the insufficient detail of the information published should however be noted. Unfortunately, the publication fails to indicate the optimal grinding fineness for each of the stages and leaves open the question on the efficiency of using $6 \mathrm{~mm}$ balls. The selection of a planetary mill as the main grinding machine for the research raises doubts as to the application prospects for the results obtained. Despite the extensive laboratory use of planetary mills over many decades, there is no information on any actually operated industrial machines of the type. The probable reason for this is the insufficient reliability of the bearing assemblies in this type of machines under high accelerations and overloads, aggravated by the difficulty of ensuring the mass balance for the material entering the grinding chambers during continuous operation of the mill in a closed cycle with a classifier.

A vibration mill is a more promising option. The reduction effects for a material crushed in a vibration mill are lower than in a planetary mill, but it has greater reliability and is manufactured in standard industrial sizes (in Russia, Germany, Japan). The specific capacity of a vibration mill when grinding metal chips is much higher and the specific energy consumption is lower than that of a conventional ball mill [8].

For the development of an energy-efficient mechanical recycling technology for metal chips to obtain a product suitable for 3D printing, it seems important to conduct further research using 
vibration grinding machines and complete the following tasks:

- establish the grinding fineness for each of the stages that would be optimal from the point of view of energy consumption distribution between the stages;

- establish the effect of the ball diameter both on the grinding of different size classes and the formation of spherical metal particles.

Preliminary tests were carried out to compile the program for such studies, select the monitoring methods and devices, and develop the experimental methods.

\section{Experiment and results}

An IVT-3 vibration bowl grinder (by Mekhanobr-Tekhnika REC) with three grinding bowls was used as the vibration grinding equipment (Fig. 2). The following three steel shot sizes were used instead of the standard grinding media:

1) with a diameter of 1.25 to $1.6 \mathrm{~mm}$ (conditionally further referred to as $\varnothing 1 \mathrm{~mm}$ ), with the bulk density of $4.26 \mathrm{~g} / \mathrm{cm}^{3}$.

2) with a diameter of 2 to $4 \mathrm{~mm}$ (conditionally further referred to as $Ø 3 \mathrm{~mm}$ ), with the bulk density of $4.54 \mathrm{~g} / \mathrm{cm}^{3}$.

3 ) with a diameter of 3 to $5 \mathrm{~mm}$ (conditionally further referred to as $\varnothing 5 \mathrm{~mm}$ ), with the bulk density of $4.37 \mathrm{~g} / \mathrm{cm}^{3}$.

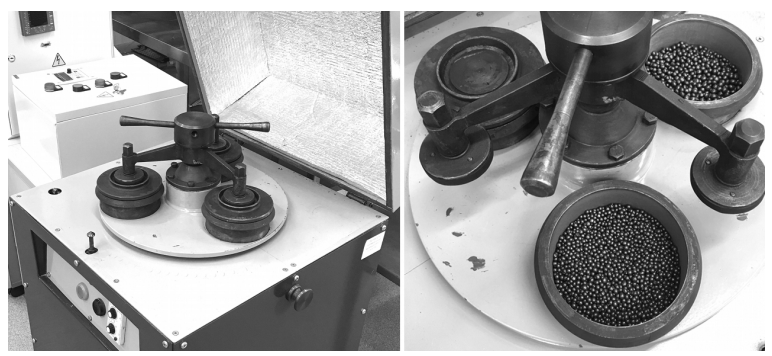

Fig. 2. IVT-3 vibration bowl grinder

The source material was represented by nickel alloy metal chips $(77 \% \mathrm{Ni})$ with the size of $100-450 \mu \mathrm{m}$, separated from the crushed product downstream of a hammer crusher.

Steel shot of a certain diameter with the equal weight was fed to each of the grinding bowls. The weight of each sample of the feed chips was $200 \mathrm{~g}$.

The volume of the ball loading during the first hour of grinding was approx. $3 / 4$ of the bowl volume or $1.4 \mathrm{~kg}$. During the next four hours of grinding $(1+4)$, the weight of the balls was reduced to $1 \mathrm{~kg}$. Then the ball loading was restored to its original value and the samples were ground for eight hours more $(1+4+8)$.

The vibration bowl grinder was operated in the discrete signal mode to prevent overheating of the bowls above $150{ }^{\circ} \mathrm{C}$ (measured as the external wall temperature of the body). After every 10 minutes of operation, the external temperature of the body of each bowl was measured using an RGK TL-80 thermal imager.

After one, five, and thirteen hours of grinding, the grinding bowls were discharged, the particle size distribution for the fraction below $450 \mu \mathrm{m}$ was established using a Microsizer 201C laser particle analyzer, and the bulk density was measured according for the size class of 100-450 $\mu \mathrm{m}$. It was not possible to measure the bulk density and effective viscosity for the size classes below $100 \mu \mathrm{m}$ due to the insufficient amount generated.

The analysis of temperature changes for the grinding bowls in the process of metal chip grinding (see Fig. 3) indicates significant differences in the temperature rise dynamics during the initial start-up and subsequent starts of the vibration grinder. In addition, a significant effect of the ball loading weight on the heating rate of the grinding bowls was established.

Since it is, indirectly, the frictional forces between the balls and the material particles that drive 
the efficiency of energy consumption for the plastic deformation of the fracture and the shape change, registration and analysis of the heat release parameters opens up prospects for developing a method for selecting the optimal volume and sizes of the ball loading for the vibromechanical spheroidization.

The high dynamics of the temperature rise in the first hour of operation for a bowl with the ball diameter of $1 \mathrm{~mm}$ is most likely due to the fact that the small diameter of the grinding medium immediately creates a large number of points of contact between the balls and the material and the resulting frictional forces ensure the conversion of energy into heat in large quantities.
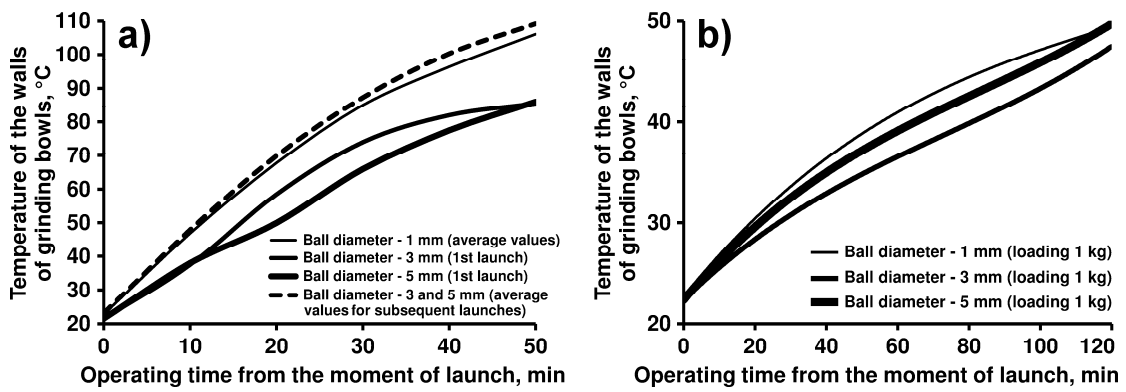

Fig. 3. Grinding bowls heating dynamics for a vibration grinder with different ball diameters:

a) with the ball weight in each bowl of $1.4 \mathrm{~kg}$ (initial and subsequent starts);

b) with the ball weight in each bowl of $1 \mathrm{~kg}$

The higher the ball diameter, the lower the heat release, which is due to the reduced number of points of contact with higher ball diameters. An increase in the number of grinding cycles did not change the temperature dynamics of bowl heating when using $1 \mathrm{~mm}$ balls, i.e. either the maximum possible number of points of contact was initially formed, or the ball weight at the given diameter was no longer sufficient for an intensive change in the shape of the material being ground. For the 3 and $5 \mathrm{~mm}$ balls, the ball loading was gradually changing the shape and size of the material, in such a way that the number of friction points was equal to or even higher than that obtained with the ball diameter of $1 \mathrm{~mm}$, which follows from the change in the nature of the curves in Fig. 3.

The analysis of the bulk density in the class of 100-450 microns confirmed the differences in the degree of transformation of the shape and size of the material being ground, depending on the size of the ball loading (see Fig. 4). When grinding with $1 \mathrm{~mm}$ balls, the material density remained unchanged all 13 hours of equipment operation. Balls with the diameter of $5 \mathrm{~mm}$ caused the most significant changes in the material being ground, with the bulk density decreasing steadily and reaching $3.13 \mathrm{~g} / \mathrm{cm}^{3}$ after 13 hours of operation.

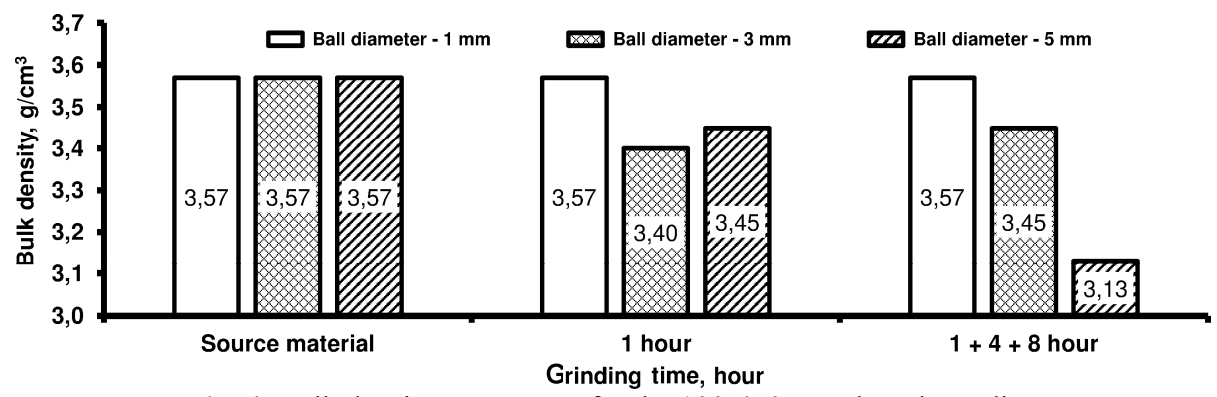

Fig. 4. Bulk density parameters for the 100-450 $\mu$ m class depending on the grinding time and the ball diameter

In order to compare the vibration grinding products with the products obtained by the traditional method, experiments were carried using a laboratory ball mill with the inner shell 
diameter of $0.3 \mathrm{~m}$ and volume of 5 liters. Grinding was carried out with the ball loading of $30 \%$ (shell volume) and the same ball diameters as for the vibration grinding. The sample weight was $300 \mathrm{~g}$ and the grinding time was 56 hours.

The grain-size composition analysis for the grinding products (see Fig. 5(a)) shows the absence of significant changes in the grain sizes when using balls with the diameters of 1 and $3 \mathrm{~mm}$ after 13 hours of operation of the vibration grinder. Consequently, 1 and $3 \mathrm{~mm}$ balls are not massive enough for the direct reduction of metal particles and their interaction with the material being ground is predominantly abrasive in nature. The content of the size class below $71 \mu \mathrm{m}$ increased by $13.5 \%$ ( 8.3 to $21.8 \%$ ), with the largest number of particles in the class below $45 \mu \mathrm{m}$ increasing by $12.5 \%$ (6.3 to $18.8 \%$ ), only after the switch to $5 \mathrm{~mm}$ balls and additional 13 hours of vibration grinding. The content of coarse classes of $315-450 \mu \mathrm{m}$ did not decrease and the reduction was only observed for the finer class of 100-315 $\mu \mathrm{m}$.

In a ball mill (Fig. 5(b)), with the grinding time 4.3 times longer and 1 and $3 \mathrm{~mm}$ balls used, there were also no significant signs of particle reduction in the particle size classes of over $160 \mu \mathrm{m}$. An increase in the number of particles below $25 \mu \mathrm{m}$ was only observed, which confirms the thesis on the predominantly abrasive nature of interactions between the material and the grinding medium with these ball sizes. Balls with the diameter of $5 \mathrm{~mm}$ produce a more significant reduction effect on coarse particles with the formation of mainly submicron particles (below $25 \mu \mathrm{m}$ ); however, as compared with vibration grinding, these results are significantly inferior in terms of the process dynamics.

This indicates that $5 \mathrm{~mm}$ balls may be used for the vibration reduction of particle sizes of over $315 \mu \mathrm{m}$ to produce particle sizes below $71 \mu \mathrm{m}$. In order to obtain intermediate particle sizes of 71-160 $\mu \mathrm{m}$, research using larger ball diameters shall be required, since the content of these size classes in the grinding product remained practically unchanged as compared with the source material.

The analysis for the fraction below $100 \mu \mathrm{m}$ found in all the final grinding products using a Bresser Science MTL-201 metallographic microscope shows the presence of a large number of 3D particles with non-lamellar shape. A more detailed study of the shape and a quantitative analysis using electron microscopy shall be required for such particles.
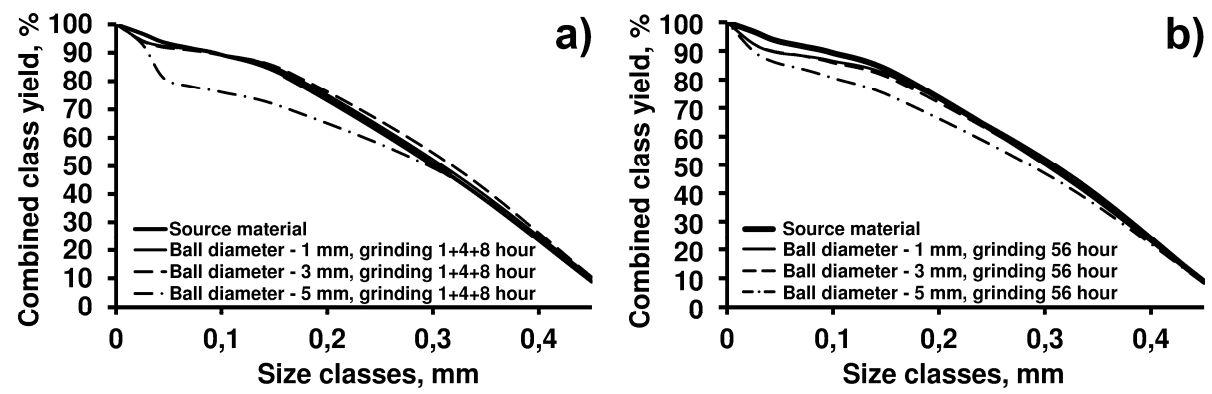

Fig. 5. Grain-size composition of grinding products at different ball diameters: a) after 13 hours in a vibration grinder; b) after 56 hours in a ball mill

\section{Conclusions}

There has been received new data on connection between parameters of balls loading and grinded particles shape. When summing up the results of the tests, the following conclusions may be drawn to establish the respective further research vector:

1) Balls with the diameters of 1 and $3 \mathrm{~mm}$ fail to produce any significant reduction effect on coarse metal particles and their interactions are predominantly abrasive in nature. The changes from lamellar to more 3D-shape for metal particles with size below $100 \mu \mathrm{m}$ in these experiments suggest that the abrasive effect may be a significant factor for the formation of the spherical shape.

2) The weight of $5 \mathrm{~mm}$ balls is sufficient to provide the vibration reduction of particles with 
the size of up to $315 \mu \mathrm{m}$ to obtain particles of less than $71 \mu \mathrm{m}$ of a non-lamellar 3D-shape.

3) Comparative grinding in a ball mill with 4.3 times longer grinding time confirmed the abrasive nature of interactions between the 1 and $3 \mathrm{~mm}$ balls and the material, with the formation of predominantly submicron particles. When using $5 \mathrm{~mm}$ balls, the reduction effect for coarse particles is higher, but the process dynamics is inferior to that observed under vibration grinding.

4) Research with larger ball diameters is required due to the absence of signs of reduction for particles of over $315 \mu \mathrm{m}$ during vibration grinding with 1-5 mm balls and the insufficient yield of the intermediate grain size classes of $71-160 \mu \mathrm{m}$. Continued research in this area will allow accurately establishing the grinding fineness for each of the stages that would be optimal in terms of energy distribution by stages.

5) For the first time a collective parameter for estimating characteristics of vibration load was proposed and piloted, namely, temperature of grinding chamber. A new ergonomic method for studying mechanical spheroidization can be developed based on the analysis of the parameter dynamics.

6) Further studies of the heat release parameters during vibration grinding of metal chips and analyses of their relationship with power consumption, ball size, ball loading level and other parameters, will open up prospects for designing a methodology to establish the optimal ball loading levels and sizes for the mechanical spheroidization process.

7) Continued research aimed at the development of the vibromechanical spheroidization technology opens the way to a significant reduction in energy consumption when recycling metal processing waste. The optimal load distribution for the two vibration grinding stages with the correct ball size selection for each stage will ensure the required spherical shape of the finely ground metal powder particles with practically the same energy consumption that has been previously required solely for grinding the chips.

\section{Acknowledgements}

The study was carried out under the grant issued by the Russian Science Foundation (Project No. 20-79-10125).

\section{References}

[1] Nizovtsev V. E., Klimov D. A., Stupenkov M. I., Bredikhina E. N. Advanced technologies for the production of powder materials for additive manufacturing. Materials of the 4th International Conference, FSUE All-Russian Research Institute of Aviation Materials, 2018, p. 203-208.

[2] Mezenin A. O., Dmitriev S. V., Cherkasova M. V. Electrical separation for the processing of mineral and technogenic raw materials. Problems and prospects of effective processing of mineral raw materials in the 21st century. Materials of the International Meeting, 2019, p. 445-448.

[3] Knyazev A. E., Vostrikov A. V. Sieving of powders in additive and granular production (review). Aviation Materials and Technologies, Vol. 11, Issue 93, 2020, p. 11-20.

[4] Grigoriev A. V., Razumov N. G., Popovich A. A., Samokhin A. V. Plasma spheroidization of powders based on Nb-Si alloys obtained by mechanical alloying. Scientific and Technical Publications of Peter the Great St.Petersburg Polytechnic University (SPbPU), Vol. 23, Issue 1, 2017, p. 247-255.

[5] Enayati M. H., Bafandeh M. R., Nosohian S. Ball milling of stainless steel scrap chips to produce nanocrystalline powder. Journal of Materials Science, Vol. 42, 2007, p. 2844-2848.

[6] Fullenwider B., Kiani P., Schoenung J. M., Ma K. From recycled machining waste to useful powders for metal additive manufacturing. The Minerals, Metals and Materials Series, 2019, https://doi.org/10.1007/978-3-030-10386-6_1.

[7] Shashanka R., Chaira D. Optimization of milling parameters for the synthesis of nano-structured duplex and ferritic stainless steel powders by high energy planetary milling. Powder Technology, Vol. 278, 2015, p. 35-45.

[8] Cherkasova M., Samukov A., Goncharov I., Mezenin A. Influence of the metal chips disintegration method on the physical and mechanical properties of metal powders obtained. Vibroengineering Procedia, Vol. 32, 2020, p. 32-37. 